\title{
THE PROJECTION PROPERTY
}

\author{
CORNEL PASNICU \\ Department of Mathematics and Computer Science, University of Puerto Rico, \\ Box 23355, San Juan, Puerto Rico 00931, USA \\ e-mail: cpasnic@upracd.upr.clu.edu
}

(Received 5 September, 2000; accepted 15 April 2001)

\begin{abstract}
In this paper we prove that the ideal property and the projection property do not conincide in general even in the separable case (despite the fact that, as we proved before, they are the same for $G A H$ algebras-and, in particular, for $A H$ algebras-and for separable $L B$ algebras). We also study the behaviour of the projection property with respect to several natural operations.
\end{abstract}

2000 Mathematics Subject Classification. 46L05, 46L99.

We begin by introducing the following definition.

Definition 1. A $C^{*}$-algebra is said to have the projection property if each of its ideals has an approximate unit consisting of projections.

In the present paper all the ideals are closed and two-sided.

In this paper we shall study the projection property: we shall prove that it differs from the ideal property (even in the separable case) - despite of a lot of "possible evidence" for the contrary conclusion - and we shall also study the behaviour of the projection property with respect to some natural operations. It is obvious that the projection property is stronger than the ideal property, whose study was suggested by G.A. Elliott. Recall that a $C^{*}$-algebra has the ideal property if each of its ideals is generated (as an ideal) by projections. The class of the $C^{*}$-algebras with the ideal property (studied in [20], [11-19]) is interesting since it contains two important classes of algebras: the real rank zero $C^{*}$-algebras ([4]) and the simple, unital $C^{*}$ algebras. Therefore, the $C^{*}$-algebras with the ideal property are very important in Elliott's classification program; (see [6]). On the other hand, in [13] we proved, in particular, that for $A H$ algebras the ideal property and the projection property coincide. We generalized this fact in [15], where we showed, in particular, that these two properties coincide for the class of $G A H$ algebras (note that a $G A H$ algebra is an inductive limit $C^{*}$-algebra $\lim _{\rightarrow} A_{n}$, where for each $n \in \mathbb{N}, A_{n}=\bigoplus_{i=1}^{k_{n}} A_{n}^{i}$ and each $A_{n}^{i}$ is a unital $C^{*}$-algebra whose proper ideals have no nonzero projections [15]; obviously, an $A H$ algebra is a $G A H$ algebra). Recently we generalized these results in the separable case, proving that the ideal property and the projection property coincide for the class of separable $L B$ algebras (see Definition 16 below and [18]). Hence, the following question is both natural and interesting: 
QUESTION 2. Do the ideal property and the projection property coincide?

The following result gives two ways of rephrasing the projecton property:

Proposition 3. Let A be a $C^{*}$-algebra. Then, the following conditions are equivalent.

(1) A has the projection property.

(2) Each ideal of $A$ is an inductive limit of unital $C^{*}$-algebras

(3) Each ideal of $A$ is an inductive limit of unital hereditary $C^{*}$-algebras of $A$.

Proof. (1) $\Rightarrow(3)$. Let $I$ be an ideal of $A$. Since $A$ has the projection property, let $\left(e_{i}\right)_{i \in \Lambda}$ be an approximate unit of projections for $I$. Now, it is not difficult to see that

$$
I=\overline{\bigcup_{i \in \Lambda} e_{i} A e_{i}}=\lim _{\rightarrow} e_{i} A e_{i}
$$

and that each $e_{i} A e_{i}$ is a unital hereditary $C^{*}$-subalgebra of $A$.

$(3) \Rightarrow(2)$ is obvious.

$(2) \Rightarrow(1)$. Let $I$ be an ideal of $A$. By hypothesis, $I=\lim \left(I_{i}, \Phi_{i, j}\right)$, where each $I_{i}$ $(i \in \Lambda)$ is a unital $C^{*}$-algebra. Since the quotient of a unital $C^{*}$-algebra is unital, we may suppose that the canonical homomorphisms $I_{i} \rightarrow I_{j}, i \leq j, i, j \in \Lambda$ and $I_{i} \rightarrow$ $I, i \in \Lambda$ are inclusions, and hence that:

$$
I=\overline{\bigcup_{i \in \Lambda} I_{i}}
$$

For each $i \in \Lambda$, let $e_{i}$ be the unit of $I_{i}$. Then, obviously $\left(e_{i}\right)_{i \in \Lambda}$ is an increasing net of projections such that for each $x \in I_{i_{0}}$ with $i_{0} \in \Lambda$, we have:

$$
x=e_{i} x\left(=x e_{i}\right), i \in \Lambda, i \geq i_{0}
$$

Hence $\left(e_{i}\right)_{i \in \Lambda}$ is an approximate unit of projections for $I$. In conclusion, $A$ has the projection property.

Proposition 4. The projection property passes to ideals and quotients and it is preserved under finite direct sums.

Proof. Obvious.

Proposition 5. Let $A$ be a $C^{*}$-algebra such that $A=\lim \left(A_{n}, \Phi_{n, m}\right)$ where each $A_{n}(n \in \mathbb{N})$ is a separable $C^{*}$-algebra with the projection property.

Then, $A$ has the projection property.

Proof. Since a quotient of a $C^{*}$-algebra with the projection property has the projection property (see Proposition 4 above), we may suppose that $A_{n} \subseteq A_{n+1}, n \in \mathbb{N}$ and that:

$$
A=\overline{\bigcup_{n \in \mathbb{N}} A_{n}}
$$



Let $I$ be an ideal of $A$. Then, by [2] it follows that $I=\overline{\bigcup_{n \in \mathbb{N}} I_{n}}$, where for each
$n \in \mathbb{N}, I_{n}:=I \bigcap A_{n}$.

For each $k \in \mathbb{N}$ let $F_{k}:=\left\{a_{m}^{k} \mid m \in \mathbb{N}\right\}$ be a dense subset of $I_{k}$. Then, obviously, $\bigcup_{k \in \mathbb{N}} F_{k}$ is a countable, dense subset of $I$. Now, since for each $n \in \mathbb{N}, I_{n}$ has an approximate unit of projections (because $A_{n}$ has the projection property and $I_{n}$ is an ideal of $A_{n}$ ), it follows that for each $n \in \mathbb{N}$ there is a projection $p_{n} \in I_{n}$ such that:

$$
\left\|a_{m}^{k}-a_{m}^{k} p_{n}\right\| \leq \frac{1}{n}, 1 \leq k, m \leq n
$$

This easily implies that for each $x \in I$ we have:

$$
x=\lim _{n} x p_{n}\left(=\lim _{n} p_{n} x\right)
$$

Now, since $I$ is separable, by [9, Theorem 6] it follows that $I$ has (a countable) approximate unit of projections. Hence, $A$ has the projection property.

QUESTION 6. Is the projection property preserved under inductive limits?

The symbol $\otimes$ will in this paper always mean the minimal tensor product (of $C^{*}$-algebras).

Recall that a $C^{*}$-algebra $A$ is exact if for all pairs $(B, J)$ of a $C^{*}$-algebra $B$ and an ideal $J$ in $B$,

$$
0 \rightarrow A \otimes J \rightarrow A \otimes B \rightarrow A \otimes(B / J) \rightarrow 0
$$

is exact ([7]). It is well-known that nuclear $C^{*}$-algebras are exact. For more information about exact $C^{*}$-algebras see e.g. [21].

We shall need in the sequel the following theorem of Kirchberg:

Theorem 7. (Kirchberg, [8, Proposition 2.13]). Let $A$ and $B$ be $C^{*}$-algebras of which at least one is exact. Then each closed two-sided ideal $K$ of the minimal tensor product $A \otimes B$ is generated by the family of rectangular ideals $\left\{I_{\alpha} \otimes J_{\alpha}\right\}_{\alpha \in I}$ contained in $K$.

As a corollary to Kirchberg's theorem we obtain:

Corollary 8. Let $A$ be a $C^{*}$-algebra with the projection property and let $B$ be a simple $C^{*}$-algebra with the projection property. If either $A$ or $B$ is exact, then $A \otimes B$ has the projection property.

Recall that an $A F$ algebra is an inductive limit $C^{*}$-algebra $\lim A_{n}$ where each $A_{n}(n \in \mathbb{N})$ is a finite dimensional $C^{*}$-algebra ([2]).

COROllary 9. Let $A$ be a $C^{*}$-algebra with the projection property and let $B$ be a simple AF algebra. Then $A \otimes B$ has the projection property.

Proposition 10. Let $A$ be a separable $C^{*}$-algebra with the projection property and let $B$ be an $A F$ algebra. Then, $A \otimes B$ has the projection property. 
Proof. Combine Proposition 4 with Corollary 9 and Proposition 5

QUESTION 11. Is the projection property preserved under forming minimal tensor products?

The answer to the above question is "no" and it follows immediately from our joint paper with M. Rørdam [19]. Indeed, in [19] we proved that $C \otimes B(H)$ does not have the ideal property, where $\mathrm{C}$ is a certain unital, simple $C^{*}$-algebra constucted by M. Dadarlat in [5] and $B(H)$ is the $C^{*}$-algebra of all the bounded, linear operators on a separable, infinite dimensional Hilbert space $H$. Then, obviously, $C \otimes B(H)$ does not have the projection property while both $C$ and $B(H)$ have the projection property.

QUeSTION 12. Is it always true that if the tensor product of $C^{*}$-algebras $A \otimes B$ has the projection property then $A$ and $B$ are $C^{*}$-algebras with the projection property?

QUESTION 13. Is the projection property preserved under stable isomorphism?

If $\mathrm{A}$ is a $C^{*}$-algebra, we shall denote by $\mathcal{P}(\mathrm{A})$, the set of all the projections of $A: \mathcal{P}(A)=\left\{p \in A \mid p=p^{*}=p^{2} \in A\right\}$.

Recall that an extension of $C^{*}$-algebras

$$
0 \rightarrow I \rightarrow A \rightarrow A / I \rightarrow 0
$$

is called quasidiagonal if there is an approximate unit $\left(p_{n}\right)_{n=1}^{\infty}$ of $I$ consisting of projections, which is quasicentral in $A$, i.e.

$$
\lim _{n \rightarrow \infty}\left\|a p_{n}-p_{n} a\right\|=0
$$

for all $a \in A$. This definition goes back to G.J. Murphy and N. Salinas.

While the projection property doesn't pass to hereditary $C^{*}$-subalgebras - as it follows from [4, Theorem 2.6] - one can prove the following two results:

Proposition 14. Let $A$ be a separable $C^{*}$-algebra such that for each ideal I of $A$, the canonical extension:

$$
0 \rightarrow I \rightarrow A \rightarrow A / I \rightarrow 0
$$

is quasidiagonal (here the map $I \rightarrow A$ is the canonical inclusion and the map $A \rightarrow A / I$ is the canonical surjection). Let $B$ be a simple, separable $C^{*}$-algebra with the projection property. Suppose that either $A$ or $B$ is exact. Then each hereditary $C^{*}$-subalgebra of $A \otimes B$ with an approximate unit of projections has the projection property.

Proof. First, let us prove that $A \otimes B$, has the same property as $A$, i.e. for each ideal $J$ of $A \otimes B$, the canonical extension:

$$
0 \rightarrow J \rightarrow A \otimes B \rightarrow(A \otimes B) / J \rightarrow 0
$$


is quasidiagonal. To start the proof, note that by the above mentioned theorem of Kirchberg (i.e. Theorem 7) it follows that $J=I \otimes B$, where $I$ is an ideal of $A$. Since the canonical extension:

$$
0 \rightarrow I \rightarrow A \rightarrow A / I \rightarrow 0
$$

is quasidiagonal (by hypothesis), let $\left(e_{n}\right)_{n \in \mathbb{N}}$ be an approximate unit of projections for $I$ which is quasicentral in $A$. Since $B$ is separable and has the projection property, by [9, Theorem 6] it follows that $B$ has an approximate unit of projections $(f)_{n \in \mathbb{N}}$. Then, it is easy to see that $\left(e_{n} \otimes f_{n}\right)_{n \in \mathbb{N}}$ is an approximate unit of projections for $I \otimes B$ which is quasicentral in $A \otimes B$. In conclusion, the above extension ( $*$ ) is quasidiagonal.

The remaining part of the proof is inspired by the proof of [16, Theorem 4.4] and by [16, Remark 4.5].

(1) First, we shall prove that $e(A \otimes B) e$ has the projection property for each $e \in \mathcal{P}(A \otimes B)$. Let $J$ be an ideal of $e(A \otimes B) e$. Then, by [10, Theorem 3.2.7], it follows that there is an ideal $I$ of $A \otimes B$ such that $J=I \cap e(A \otimes B) e$. But, it is easy to see that $J=e I e$. Now, since the canonical extension:

$$
0 \rightarrow I \rightarrow A \otimes B \rightarrow(A \otimes B) / I \rightarrow 0
$$

is quasidiagonal by our above discussion, it follows by [12, Lemma 3.7 (1)] that the "reduced" canonical extension:

$$
0 \rightarrow e I e \rightarrow e(A \otimes B) e \rightarrow(e(A \otimes B) e) / e I e \rightarrow 0
$$

is quasidiagonal. In particular, $J=e$ Ie has a countable approximate unit of projections. Hence $e(A \otimes B) e$ has the projection property.

(2) Now we shall prove the general case. Let $C$ be a hereditary $C^{*}$-subalgebra of $A \otimes B$ with an approximate unit of projections. Because $C$ is also separable (since $A \otimes B$ is separable), by [9, Theorem 6] it follows that $C$ has an approximate unit $\left(e_{n}\right)_{n \in \mathbb{N}}$ of projections.

Then, it is not difficult to see that:

$$
C=\overline{\bigcup_{n \in \mathbb{N}} e_{n}(A \otimes B) e_{n}}=\lim _{\rightarrow} e_{n}(A \otimes B) e_{n}
$$

Note that by (1), each $e_{n}(A \otimes B) e_{n}$ has the projection property. Since also any $C^{*}$-algebra $e_{n}(A \otimes B) e_{n}$ is separable, by Proposition 5 it follows that $C$ has the projection property.

Let us recall some definitions from [18], which will be needed in the sequel:

Definition 15 ([18]). $A$ is called a basic $C^{*}$-algebra if $A$ is a unital $C^{*}$-algebra such that each of its ideals generated (as ideals) by projections is a direct summand of $A$.

Note that any finite direct sum of unital $C^{*}$-algebras whose proper ideals have no non-zero projections is a basic $C^{*}$-algebra. 
Definition 16 ([18]). Let $A$ be a $C^{*}$-algebra. Then $A$ is called an LB algebra if : for each $\epsilon>0$, each $n \in \mathbb{N}$ and each $x_{1}, x_{2}, \ldots, x_{n} \in A$, there are a basic $C^{*}$-algebra $B$, $a *$-homomorphism $\Phi: B \rightarrow A$ then $\tilde{x}_{1}, \tilde{x}_{2}, \ldots, \tilde{x}_{n} \in B$ satisfying

$$
\left\|\Phi\left(\tilde{x}_{k}\right)-x_{k}\right\|<\epsilon, \quad 1 \leq k \leq n
$$

and such that if $x_{k} \in \mathcal{P}(A)$ for some $1 \leq k \leq n$, then $\tilde{x}_{k} \in \mathcal{P}(B)$.

Note that each $C^{*}$-algebra which is an inductive limit of basic $C^{*}$-algebras is an $L B$ algebra. In particular, each $A H$ algebra or more generally, each $G A H$ algebra ([15]) is an $L B$ algebra. Recall that a $G A H$ algebra is an inductive limit $C^{*}$-algebra $\lim A_{n}$, where each $A_{n}(n \in \mathbb{N})$ is a finite direct sum of unital $C^{*}$-algebras whose

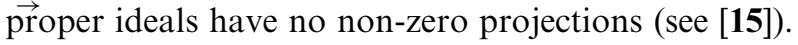

TheORem 17. Let $A$ be separable LB algebra with the ideal property and let $B$ be a simple, separable $C^{*}$-algebra with the projection property. If either $A$ or $B$ is exact, then each hereditary $C^{*}$-subalgebra of $A \otimes B$ with an approximate unit of projections has the projection property.

Proof. Note that by [18] it follows that for each ideal $I$ of $A$, the canonical extension:

$$
0 \rightarrow I \rightarrow A \rightarrow A / I \rightarrow 0
$$

is quasidiagonal. Now, the proof follows using also Proposition 14.

REMARKS 18. (1) Note that the projection property is not preserved under extensions. Indeed, we constructed jointly with $\mathrm{M}$. Dadarlat in [14, Theorem 5.1] extensions $0 \rightarrow I \rightarrow A \rightarrow B \rightarrow 0$ where $I$ and $B$ are simple $A H$ algebras (in particular, $I$ and $B$ are $C^{*}$-algebras with the projection property) and $A$ does not have the ideal property and hence, in particular, $A$ does not have the projection property.

(2) The projection property is not preserved under homotopy equivalence (and hence under shape equivalence, in the separable case). Indeed, $C([0,1])$ and $\mathbb{C}$ are homotopy equivalent (since $[0,1]$ is contractible), $\mathbb{C}$ obviously has the projection property while $C([0,1])$ has not the projection property (since its non-zero ideals do not contain non-zero projections).

Finally, we give the answer to most of the above natural questions:

THEOREM 19. The above Questions 2, 12 and 13 have negative answers even in the separable case.

Proof. (1) Let $M$ be a separable, simple $C^{*}$-algebra with a countable approximate unit of projections and a non-zero real rank. Then, by [4, Theorem 2.6] there is a (separable) hereditary $C^{*}$-subalgebra $A$ of $M$ which does not have an aproximate unit of projections. Since $M$ is simple, it follows by [10, Theorem 3.2.8] that $A$ is also a simple $C^{*}$-algebra. Now, using the fact that $M$ is separable and simple, by a result of L.G. Brown [3, Theorem 2.8] it follows that $M$ and $A$ are stably isomorphic: 
$A \otimes \mathcal{K} \cong M \otimes \mathcal{K}$, where $\mathcal{K}$ is the $C^{*}$-algebra of all compact operators on a separable, infinite dimensional Hilbert space. But $A \otimes \mathcal{K}$ is simple (since $\mathcal{A}$ and $\mathcal{K}$ are simple) and it has a countable approximate unit of projections since $M \otimes \mathcal{K}$ has (let $\left(e_{n}\right)_{n \in \mathbb{N}}$ (resp. $\left.\left(f_{n}\right)_{n \in \mathbb{N}}\right)$ be an approximate unit of projections of $M(\operatorname{resp} . \mathcal{K})$; then $\left(e_{n} \otimes f_{n}\right)_{n \in \mathbb{N}}$ is clearly an approximate unit of projections for $M \otimes \mathcal{K}$ ). In conclusion, $A \otimes \mathcal{K}$ has the projection property while $A$ has not. This proves that Question 12 has a negative answer even in the separable case (take $B=\mathcal{K}$ ) and also that Question 13 has a negative answer even in the separable case.

(2) Let $A$ be a separable, simple $C^{*}$-algebra, with the real rank different from 0 and such that each non-zero hereditary $C^{*}$-subalgebra contains a non-zero projection (note that B. Blackadar and A. Kumjian constructed in [1] an $A H$ algebra with these properties). Since the real rank of $A$ is not zero, by [4, Theorem 2.6] it follows that there is a non-zero hereditary $C^{*}$-subalgebra $B$ of $A$ which does not have an approximate unit of projections. On the other hand, since $A$ is simple, by a general argument (see e.g. [10, Theorem 3.2.8]) it follows that $B$ is simple. Hence $B$ has not the projection property. But, by construction, $B$ contains a non-zero projection $p$. Since the $C^{*}$-algebra $B$ is simple, it follows that the (closed, two-sided) ideal generated in $B$ by $p$ is $B$. Hence, $B$ has the ideal property, is separable and has not the projection property. This gives a negative answer to Question 2 even in the separable case.

Acknowledgements. We are indebted to M. Dadarlat and to N.C. Phillips for useful conversations related to the subject of this paper. This material is based upon work supported by, or in part by, the U.S. Army Research Office under grant number DAAD19-00-1-0152. This research was partially supported also by NSF grant DMS-9622250.

\section{REFERENCES}

1. B. Blackadar and A. Kumjian, Skew products of relations and the structure of simple $C^{*}$-algebras, Math. Z. 189 (1985), 55-63.

2. O. Bratteli, Inductive limits of finite dimensional $C^{*}$-algebras, Trans. Amer. Math. Soc. 171 (1972), 195-234.

3. L. G. Brown, Stable isomorphism of hereditary subalgebras of $C^{*}$-algebras, Pacific $J$. Math. 71 (1977), 335-348.

4. L. G. Brown and G. K. Pedersen, $C^{*}$-algebras of real rank zero, J. Funct. Anal. 99 (1991), 131-149.

5. M, Dadarlat, Nonnuclear subalgebras of AF algebras, Amer. J. Math. 122 No 3, (2000), 581-597.

6. G. A. Elliot, The clasification problem for amenable $C^{*}$-algebras, in Proceedings of the International Congress of Mathematicians, Vol. 1, 2 (Birkhäuser, Basel, 1995), 922-932.

7. E. Kirchberg, Positive maps and $C^{*}$-nuclear algebras, Proc. International Conference on Operator Algebras, Ideals and their Applications in Theoretical Physics, Leipzig, September 12-20, 1977 (Teubner texte, Leipzig, 1978), 255-257.

8. E. Kirchberg, The classification of purely infinite $C^{*}$-algebras using Kasparov's theory, Fields Institute Communication series, to appear.

9. G. J. Murphy, Diagonality in $C^{*}$-algebras, Math. Z. 199 (1988), 279-284.

10. G. J. Murphy, $C^{*}$-algebras and operator theory (Academic Press, 1990).

11. C. Pasnicu, $A H$ algebras with the ideal property in Operator algebras and operator theory, Contemporary Mathematics, Vol. 228 (A.M.S., Providence, Rhode Island, 1998), 277288.

12. C. Pasnicu, Extensions of $A H$ algebras with the ideal property, Proc. Edinburgh Math. Soc. (2) 42 (1999), 65-76. 
13. C. Pasnicu, Shape equivalence, nonstable $K$-theory and $A H$ algebras, Pacific J. Math. 192 (2000), 159-182.

14. C. Pasnicu, On the $A H$ algebras with the ideal property, J. Operator Theory 43 (2000), 389-407.

15. C. Pasnicu, Ideals generated by projections and inductive limit $C^{*}$-algebras, Rocky Mountain J. Math. 37, 3 (2001), 1083-1095.

16. C. Pasnicu, On the (strong) GAH algebras, Rev. Roumaine Math. Pures Appl. 227, 1 (2001), 127-132.

17. C. Pasnicu, The ideal property and traces, Math. Nachr., to appear.

18. C. Pasnicu, $L B$ algebras, J. Operator Theory, to appear.

19. C. Pasnicu and M. Rørdam, Tensor products of $C^{*}$-algebras with the ideal property, J. Funct. Anal. 177 (2000), 130-137.

20. K. H. Stevens, The classification of certain non-simple approximate inverval algebras, $\mathrm{Ph}$. D. thesis (University of Toronto, 1994).

21. S. Wassermann, Exact $C^{*}$-algebras and related topics, Lecture Notes Series, Vol. 19, Seoul National University, Research Institute of Mathematics, Global Analysis Research Center, Seoul, Korea, 1994. 